\title{
Ebp1-mediated inhibition of cell growth requires serine 363 phosphorylation
}

\author{
DAMILOLA AKINMADE ${ }^{1,2}$, MYOUNGHEE LEE ${ }^{2}$, YUEXING ZHANG ${ }^{1,2}$ and ANNE W. HAMBURGER ${ }^{1,2}$ \\ ${ }^{1}$ Department of Pathology, ${ }^{2}$ Greenebaum Cancer Center, University of Maryland, Baltimore, MD, USA
}

Received April 2, 2007; Accepted May 14, 2007

\begin{abstract}
Ebp1 is an ErbB3 binding phosphoprotein with pleiotropic effects. Overexpression of Ebp1 represses transcription of E2F1 responsive cell cycle regulated genes and inhibits cell growth. However, the effect of phosphorylation on Ebp1-mediated transcriptional repression and cell growth inhibition is currently unknown. In this study, we show that serine 363 (S363) of Ebp1 is phosphorylated in vivo. Although total Ebp1 is located in the nucleus, organelles and the cytoplasm, Ebp1 phosphorylated at S363 (Ebp1 pS363) is localized exclusively to the nucleus. Mutation of S363 to alanine did not change the subcellular localization of Ebp1. However, the S363A mutation significantly decreased the ability of Ebp1 to repress transcription and abrogated its ability to inhibit cell growth. We have previously shown that Ebp1 can bind the E2F1 promoter in vitro and in vivo as part of a protein complex and that Ebp1-transcriptional repression is mediated via its interaction with the co-repressors HDAC2 and $\mathrm{mSin} 3 \mathrm{a}$ present in this complex. Although Ebp1 S363A interacted with an E2F1 promoter element, it did not bind HDAC2 and mSin3a. These results indicate the importance of S363 phosphorylation in the function of Ebp1.
\end{abstract}

\section{Introduction}

ErbB3 (HER3), a member of the epidermal growth factor receptor (EGFR) family, is a key regulator of cell growth and differentiation (1). ErbB3 lacks kinase activity $(2,3)$, necessitating its interactions with other proteins to exert its biological effects. ErbB3 preferentially heterodimerizes with ErbB2 after binding its cognate ligand heregulin (HRG). This pairing results in potent receptor transactivation, leading to activation of mitogen activated protein kinase (MAPK), protein $\mathrm{B}$ (AKT) and phosphotidyl inositol 3 kinase (PI3K) pathways

Correspondence to: Dr A.W. Hamburger, Greenebaum Cancer Center, University of Maryland, BRB 9-029, 655 W. Baltimore Street, Baltimore, MD 21201, USA

E-mail: ahamburg@som.umaryland.edu

Key words: Ebp1, PA2G4, phosphorylation, gene expression, cell growth, DNA binding
$(4,5)$. ErbB3 mutation or overexpression results in malignancies of the breast, prostate, brain and liver $(1,6-8)$.

An ErbB3 binding protein (Ebp1) was isolated in our laboratory during a yeast two-hybrid screen for ErbB3 interacting proteins (9). Ebp1 is identical to the murine p38-2G4 protein which was isolated in a screen for DNA binding proteins (10). Ebp1 is highly conserved throughout evolution and the potato homologue has activity in human cells (11). Ebp1 is highly expressed in various mammalian cell lines except in the non-transformed MCF10A breast cell line. Ebp1 is also expressed in normal liver, brain, prostate and breast tissues $(12,13)$. Ebp1 inhibits cell growth both in vivo (14) and in vitro $(15,16)$, but also prevents apoptosis in neuronal cells (17). Overexpression of Ebp1 inhibits the transcription of reporter genes controlled by cyclin D, cyclin E and c-myc promoters and the transcription of endogenous E2F1 and c-myc genes via its binding to an E2F1 consensus element (18-20). Overexpression of Ebp1 in breast cancer cells inhibits cell growth, while promoting G2/M cell cycle arrest and cellular differentiation (15). An Ebp1 mutant lacking the last 72 amino acids (aa 322-394) is unable to either repress transcription or suppress cell growth (18), suggesting that the C terminal domain is critical for Ebp1's growth inhibitory function. This domain directly binds active histone deacetylase 2 (HDAC2) and indirectly binds the E2F1 promoter through a complex which includes Rb, HDAC2, mSin3a and E2F1 $(19,20)$. Treatment with the ErbB3 ligand HRG increases binding of Ebp1 to the E2F1 promoter complex and enhances Ebp1mediated repression of E2F1 regulated gene transcription (20). Recent data also suggest that Ebp1 is an RNA binding protein $(16,21,22)$.

Previous studies in our laboratory have demonstrated, via orthophosphate labeling of whole cells, that Ebp1 is a phosphoprotein (23). As the biologically important $\mathrm{C}$ terminal domain contains several putative phosphorylation sites, we examined the role that phosphorylation plays in Ebp1 function. Using a phospho-specific antibody, we found that the S363 residue of Ebp1, found in the $\mathrm{C}$ terminal domain, is phosphorylated in vivo. Ebp1 phosphorylated at S363 is localized exclusively to the nucleus. Mutation of this site to alanine (S363A) disrupts the ability of Ebp1 to repress gene transcription and inhibit cell growth. Although the mutation did not affect Ebp1 binding to an E2F1 promoter consensus element, it altered the ability of Ebp1 to bind the transcriptional co-repressors HDAC2 and mSin3a. These studies indicate the importance of S363 phosphorylation in Ebp1 function. 


\section{Materials and methods}

Cell culture and transfections. AU565, MCF7, MCF10A and COS7 cells were obtained from the American Type Culture Collection (Manassas, VA) and maintained at $37^{\circ} \mathrm{C}$ in a humidified atmosphere of $5 \% \mathrm{CO}_{2}$ in air. AU565 and MCF7 cells were cultured in RPMI-1640 (Biofluids, Rockville, MD), MCF10A cells were cultured in MEGM complete media (Cambrex, East Rutherford, NJ) supplemented with $100 \mathrm{ng} / \mathrm{ml}$ Cholera toxin (Calbiochem, San Diego, CA) and COS7 cells were cultured in DMEM/F-12 (Biofluids). All media (except MEGM) were supplemented with $10 \%$ fetal bovine serum (Sigma, St. Louis, MO) and 1\% penicillin/streptomycin except where noted. Cells were transfected using Optimem I media (Invitrogen, Carlsbad, CA) and Fugene 6 mammalian transfection reagent (Roche, Indianapolis, IN) according to the manufacturer's instructions.

Reagents. Heregulin $\beta 1$ (HRGß1) was obtained from R\&D Systems Inc. (Minneapolis, MN) and Geneticin (G418) from Invitrogen.

Plasmids. Ebp1 has three putative start sites ATG\#1 (p52, U59435, start site at nucleotide 98), ATG\#2 (p48, start site at nucleotide 164) and ATG\#3 (p38, start site at nucleotide 254) $(18,24)$. The EGFP-Ebp1 p52 and CMV10-Ebp1 p52 plasmids were constructed by cloning full-length Ebp1 into the BamHI/ EcoRI sites of the EGFP-C1 vector (Clontech, Palo Alto, CA) and the EcoRI/BamHI sites of the CMV10 vector (Sigma) respectively.

Site-directed mutagenesis. The S363A mutation was created in EGFP-Ebp1 and CMV10-Ebp1 using Stratagene's QuikChange II XL site-directed mutagenesis kit (La Jolla, CA). The forward primer (5'CTCCTCCAGAGTTCTGCAGCTCGA AAAACCCAGAAAAAG) was reverse complemented to obtain the reverse primer. All primers were synthesized at the Biopolymer Core Lab at University of Maryland, Baltimore (BCL-UMB).

Automated fluorescence sequencing primers. Sequencing primers for the CMV10 based plasmids (CMV24 and CMV30) were obtained from Sigma. For the EGFP-C1 based plasmids, the EGFPC primer (Clontech) and the EGFP-C1 3' primer (5'GGGGGAAGTGTGGGAGGTTTTTTAAAGC, BCLUMB) were used. Samples were sequenced at the BCLUMB.

Phospho-Ebpl antibody development. Polyclonal phospho S363 Ebp1 antibody (pS363-Ebp1) was custom-made at Invitrogen by injecting rabbits with a synthetic phosphopeptide corresponding to amino acid residues ${ }^{357}$ LLQSSApSRKTQKK. Serum collected from the rabbits was affinity purified using the synthetic phosphopeptide. The unphosphorylated peptide LLQSSASRKTQKK (Peptide 1) and phosphorylated peptide LLQSSApSRKTQKK (Peptide 2) were used to analyze the affinity-purified antibodies via indirect ELISA.

Peptide neutralization assay. Peptides 1 and $2(2.5 \mathrm{mg} / \mathrm{ml}$, Invitrogen) were combined separately with the affinity-purified antibody in a 50:1 mass ratio. The mixture was diluted 1:4000 in 5\% bovine serum albumin (BSA) and 5\% non-fat milk (1:1). PVDF blots with $10 \mu \mathrm{g}$ of AU565 lysates per lane were incubated with the primary antibody/peptide mixture and probed with a goat anti-rabbit HRP secondary antibody. Protein bands were visualized using an ECL detection kit from Pierce (Rockford, IL).

Dephosphorylation. Plasmid DNA, cell lysates or IP eluates were dephosphorylated with $100 \mathrm{U}$ total calf intestinal alkaline phosphatase (CIAP, Promega, Madison, WI) at $37^{\circ} \mathrm{C}$ for $1 \mathrm{~h}$. Reactions were heat-inactivated at $65^{\circ} \mathrm{C}$ for $10 \mathrm{~min}$. DNA reactions were cleaned with the QIAEX II gel extraction kit (Qiagen, Valencia, CA). Protein reactions were run directly on SDS gels.

Western blot assay. Total cell lysates were prepared by direct lysis with NTEN lysis buffer $(20 \mathrm{mM}$ Tris- $\mathrm{HCl}$ pH 8.0, $150 \mathrm{mM} \mathrm{NaCl}, 1 \mathrm{mM}$ EDTA, $0.5 \%$ NP-40, $10 \%$ glycerol). Protein concentrations were determined using the BioRad detergent compatible protein assay kit. The samples were mixed with Laemmli sample buffer and resolved by SDSPAGE. Proteins were transferred to PVDF membranes and immunoblotted with the appropriate primary and secondary antibodies. An enhanced chemiluminescent (ECL) detection kit (Pierce) was used to visualize the bands.

Antibodies. Primary antibodies included Ebp1 (rabbit, Upstate), pS363-Ebp1 (rabbit, Invitrogen), Flag M2 (mouse, Sigma), GFP (mouse, Clontech), Actin (mouse or rabbit, Sigma), Paxillin (mouse, BD Transduction Laboratories, San Jose, CA), Grp 94 (rat, StressGen, San Diego, CA), mSin3a (rabbit, Santa Cruz Biotechnology, Santa Cruz, CA), HDAC2 (rabbit, Zymed, South San Francisco, CA). Secondary antibodies included goat anti-rabbit HRP (BioRad), goat anti-rat HRP (KPL, Gaithersburg, MD) and sheep anti-mouse HRP (Amersham, Piscataway, $\mathrm{NJ}$ ).

Immunoprecipitation. Ebp1 was immunoprecipitated from MCF7 cell lysates using an Ebp1 antibody (Upstate, Lake Placid, NY) following the Seize-X Immunoprecipitation kit protocol (Pierce). Flag-Ebp1 was immunoprecipitated from MCF7 cell lysates using anti-Flag M2 Agarose beads (Sigma) following the manufacturer's protocol. The immunoprecipitated proteins were resolved by SDS-PAGE and analyzed by Western blotting.

Serum starvation and heregulin treatment. AU565 cells were grown in 100-mm dishes with RPMI-1640 media containing $10 \% \mathrm{FBS}$ and $1 \%$ penicillin/streptomycin until confluent. The cells were rinsed with PBS and the medium was replaced with RPMI-1640 containing $0.1 \%$ FBS. The cells were then serum-starved for $48 \mathrm{~h}$ after which the medium was aspirated and cells subjected to differential detergent fractionation (DDF, described below). For heregulin treatment, the above protocol with the following changes was applied: after the 48-h serum starvation, $10 \mathrm{ng} / \mathrm{ml} \mathrm{HRGß1}$ was added and the plates were incubated for an additional $1.5 \mathrm{~h}$, after which cells were subjected to DDF.

Differential detergent fractionation. Confluent plates of AU565 cells were fractionated according to the method of Rambsy and 
Makowski (25) in the following manner: For the cytoplasmic fraction, 100-mm plates were incubated with $500 \mu 1$ Digitonin/ EDTA extraction buffer and rocked gently on ice for $5 \mathrm{~min}$. The resulting cytoplasmic lysates were transferred into a clean 1.5-ml microfuge tube. For the organelle fraction, the same plates were then incubated with $500 \mu 1$ Triton X-100 extraction buffer and rocked gently on ice for $10 \mathrm{~min}$. Organelle lysates were transferred into a clean 1.5-ml microfuge tube. For the nuclear fraction, the same plates were incubated with $250 \mu 1$ Tween-40/DOC extraction buffer and rocked gently on ice for $5 \mathrm{~min}$. Nuclear lysates were transferred into a clean 1.5-ml microfuge tube. Lysates were resolved by SDS-PAGE and analyzed by Western blotting.

Immunofluorescence and GFP fluorescence. For immunofluorescence analysis, AU565 cells were grown on glass cover slips in 35-mm dishes to 50-80\% confluency overnight. Cells were rinsed with $0.1 \%$ TBST, fixed with $1: 1$ methanol/ acetone for $2 \mathrm{~min}$ at RT, rinsed again and blocked with $10 \%$ FBS in $0.1 \%$ TBST for $1 \mathrm{~h}$. Cells were then rinsed and incubated with non-specific rabbit IgG, HDAC2 and pS363Ebp1 primary antibodies (see above). Cells were rinsed and incubated with fluorescein isothiocyanate (FITC) conjugated secondary antibodies (KPL). Cells were rinsed and the coverslips were mounted upside down onto slides using Vectashield mounting media (Vectorlabs, Burlingame, CA) containing $1.5 \mu \mathrm{g} / \mathrm{ml}$ DAPI. The coverslip perimeter was sealed with clear nail varnish. Immunofluorescence was visualized using a Carl Zeiss Axiovert 200 microscope and images were captured using the attached digital AxioCam HR and analyzed with the AxioVision digital imaging software. For GFP fluorescence data, MCF7 cells stably transfected with GFP-C1, GFP-Ebp1 or GFP-Ebp1 S363A plasmids were grown on glass coverslips as described above. GFP fluorescence was viewed without fixation and images were captured as in the immunofluorescence method.

Dual luciferase assay. MCF7 cells (5x104/well) were transfected with $0.5 \mu \mathrm{g}$ of CMV10, CMV10-Ebp1 or CMV10-Ebp1 S363A plasmids using Fugene 6. Each vector was assigned 8 wells. pE2F1-luc $(0.5 \mu \mathrm{g})$ (a firefly luciferase reporter gene under the control of the -225 to +1 region of the E2F1 promoter) (26) and $5 \mathrm{ng}$ of pRL-TK vector (a Renilla luciferase reporter gene under the control of the thymidine kinase promoter) were co-transfected in each well, the latter included as an internal control. Forty-eight hours after transfection, cells were lysed and luciferase activity determined using a dualluciferase reporter assay (Promega). The activities of Renilla luciferase were used to normalize any variations in transfection efficiency. The data are expressed as relative light units (RLU) which is the ratio of E2F1-luc RLU:pRL-TK RLU for each sample.

DNA affinity precipitation. On the day after reaching $80 \%$ confluency, MCF7 cells were transfected with $4 \mu \mathrm{g}$ of GFP-C1, GFP-Ebp1 or GFP-Ebp1 S363A plasmids. Cells were selected with G418 $(500 \mu \mathrm{g} / \mathrm{ml})$. Cells were lysed with NTEN buffer containing protease and phosphatase inhibitors (also used as wash buffer) and lysates used for DNA affinity precipitation. Twenty $\mu \mathrm{g}$ of biotin modified wild-type E2F1 -35 to +1 promoter sequence (consensus motif in bold: GGCTCTTTC GCGGCAAAAAGGATTTGGCGCGTAAAA) (20) was coupled to $500 \mu 1$ of pre-washed streptavidin Magnasphere Paramagnetic particles (Promega) for $10 \mathrm{~min}$ at RT. The coupled oligonucleotide (oligo) was washed twice and incubated with $700 \mu \mathrm{g}$ of cell lysate and $10 \mu \mathrm{g}$ of salmonsperm DNA. The mixture was brought up to $1 \mathrm{ml}$ with the wash buffer and incubated for $2 \mathrm{~h}$ at $4^{\circ} \mathrm{C}$. The precipitates were washed once, then resolved by SDS-PAGE and analyzed by Western blotting.

Colony inhibition assays. MCF7 cells were seeded into 12-well plates at $1 \times 10^{4}$ cells/well and cultured in complete media. Cells were transfected with $2 \mu \mathrm{g}$ of GFP-C1, GFP-Ebp1 or GFP-Ebp1 S363A plasmids. Each vector was assigned 6 wells. After three weeks of selection with G418 $(1100 \mu \mathrm{g} / \mathrm{ml})$, the plates were stained with crystal violet and the number of surviving colonies was counted.

Statistical analysis. Data were analyzed using a two-tailed Student's t-test with Microsoft Excel. Differences with a $\mathrm{p}<0.05$ were deemed significant.

\section{Results}

Specificity of the pS363-Ebpl antibody. The last 72 amino acids in the $\mathrm{C}$ terminal of Ebp1 are critical to its normal function (18). This domain also contains a putative nuclear localization signal ${ }^{368}$ KKKKKK. Phosphorylation of phosphoresidues surrounding a nuclear localization signal can alter the subcellular distribution and function of a protein (27). Therefore, we generated a phospho-specific antibody against a predicted phosphorylated serine site (S363) and tested the antibody's specificity for Ebp1 phosphorlated at serine 363 (Ebp1 pS363). The antibody was pre-incubated with either a phosphorylated peptide $\left({ }^{357}\right.$ LLQSSApSRKTQKK) or an unphosphorylated peptide ( ${ }^{357}$ LLQSSASRKTQKK) and used in Western blot analysis of lysates from logarithmically growing AU565 estrogen receptor negative breast cancer cells. AU565 cells were used as we previously found, by orthophosphate labeling, that Ebp1 is phosphorylated in this cell line (23). The antibody detected a band at $48 \mathrm{kDa}$ as previously reported for endogenous Ebp1 $(12,16,17)$. Recognition of endogenous Ebp1 pS363 by the antibody was abolished when the antibody was pre-incubated with the phosphopeptide (Fig. 1A, upper panel). Ebp1 was present in both lanes when the blot was probed with an anti-Ebp1 antibody (Fig. 1A, lower panel). To further demonstrate the specificity of the phospho-antibody, Ebp1 immunoprecipitated from MCF7 cells was dephosphorylated and resolved by SDS-PAGE. The p3653 antibody was unable to recognize dephosphorylated Ebp1 (Fig. 1b). We next tested the ability of the phospho-antibody to recognize Ebp1 that was mutated at S363. MCF7 cells were stably transfected with the CMV10 control vector, CMV10-Flag-Ebp1 or CMV10Flag-Ebp1 S363A. The cells were lysed and Flag-tagged proteins were immunoprecipitated and resolved by SDSPAGE (Fig. 1B, upper panel). The pS363-Ebp1 antibody was unable to recognize the Flag-Ebp1 S363A mutant (Fig. 1C, bottom panel). Finally, lysates of MCF10A cells, which do not express Ebp1 (12), were resolved by SDS-PAGE and 
A

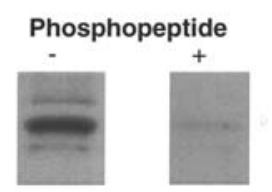

IB: pS363

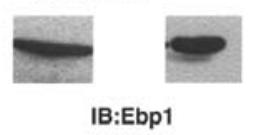

U CMV Ebp1 S363A

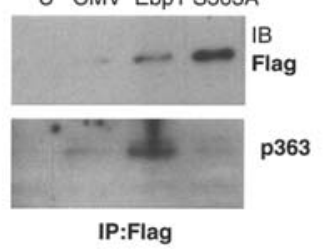

B



Ebp1

MCF7 MCF10A

D

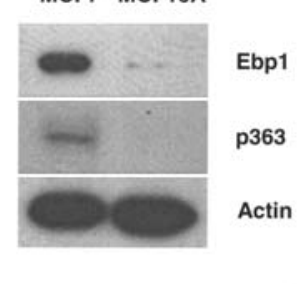

Figure 1. Validation of the Ebp1 phospho-specific antibody, pS363-Ebp1. (A) Neutralization of the pS363-Ebp1 antibody by the immunizing phosphopeptide. AU565 cell lysates were resolved on 10\% SDS gels. Blots containing the transferred proteins were analyzed using the polyclonal pS363-Ebp1 antibody pre-incubated with unphosphorylated LLQSSASRKTQKK (-) or phosphorylated peptides LLQSSApSRKTQKK (+) (top panel). The blots were also analyzed using Ebp1 antibody (bottom panel). (B) Effect of phosphatase treatment on pS363-Ebp1 antibody reactivity. Ebp1 was immunoprecipitated from AU565 lysates and treated without (-) or with (+) $100 \mathrm{U}$ total calf intestinal alkaline phosphatase (CIAP). The immunoprecipitated proteins were resolved on $10 \%$ SDS gels and the blots analyzed using pS363-Ebp1 and Ebp1 antibodies as indicated. (C) The pS363-Ebp1 antibody did not recognize Ebp1 S363A. MCF-7 cells were not transfected (U) or stably transfected with plasmids encoding the CMV10 control, a 3X Flag-tagged wild-type Ebp1, or a Flag-tagged S363A Ebp1. Cells were lysed and the fusion proteins were immunoprecipitated using anti-Flag beads. Immunoprecipitates were resolved by SDS-PAGE and analyzed by Western blotting using Flag and pS363-Ebp1 antibodies as indicated. (D) The pS363-Ebp1 antibody did not detect protein in MCF10A cell lysates. MCF7 and MCF10A lysates were resolved on 10\% SDS gels. The blots were probed for Ebp1, pS363-Ebp1, and Actin as indicated.

analyzed by Western blotting. The phospho-antibody did not detect Ebp1 pS363 in MCF10A cell lysates, but did detect pS363 in MCF7 lysates as expected (Fig. 1D).

Endogenous Ebpl phosphorylated at S363 was localized exclusively to the nucleus. In order to determine where Ebp1 pS363 was localized in the cell and if serum and heregulin treatment had any effect on its expression levels and localization, we subjected AU565 cells to differential detergent fractionation (DDF) (25). We determined the purity of the subcellular fractions using antibodies specific to each compartment (Fig. 2A). Using a polyclonal antibody to recombinant Ebp1, we found total Ebp1 in the nuclear, organelle and cytoplasmic fractions as previously determined by immunofluorescent analysis (18). Ebp1 distribution did not change with serum or heregulin treatment at the times tested (Fig. 2B and C, top panels). In contrast, Ebp1 pS363 was confined to the nucleus (Fig. 2B and C, bottom panels). Slight increases in $\mathrm{S} 363$ phosphorylation in response to serum (Fig. 2B, bottom panel) and heregulin (Fig. 2C, bottom panel) treatment were observed. The exclusive nuclear localization of Ebp1 pS363 was confirmed in AU565 cells by immunofluorescence (Fig. 2D).
Since Ebp1 pS363 was localized to the nucleus and nuclear localization can be mediated by phosphorylation (27), we determined if phosphorylated S363 was required for the nuclear localization of Ebp1. We created a S363A mutation in GFPEbp1 by site-directed mutagenesis. The alanine substitution mimics an unphosphorylatable state. MCF7 cells were transfected with GFP, GFP-Ebp1 or GFP-Ebp1 S363A plasmids. Cells were selected in G418 $(500 \mu \mathrm{g} / \mathrm{ml})$ for four weeks and then logarithmically growing cells were examined. The GFPtagged proteins were expressed at the expected molecular weight at approximately equal levels (Fig. 3A). Fluorescence imaging revealed no differences in the subcellular localization of GFP-Ebp1 S363A as compared to wild-type GFP-Ebp1 in MCF7 cells (Fig. 3B). Thus, phosphorylation at S363 was not required for nuclear localization.

Transcriptional repression by Ebpl was partially dependent on S363 phosphorylation. We next compared the biological activities of the Ebp1 S363A mutant with that of wild-type Ebp1. Ebp1 represses transcription of endogenous and exogenous E2F1 regulated genes (19). Therefore, the effect of the S363A mutation on the ability of Ebp1 to repress an exogenous reporter gene controlled by the E2F1 promoter was determined by transfecting MCF7 cells with pRL-TK, E2F1-Luc and either CMV10, CMV10-Ebp1 or CMV10-Ebp1 S363A. Both wild-type and mutant Flag-tagged proteins were intact and expressed at approximately equal levels (Fig. 4A). Forty-eight hours after transfection, the dual luciferase reporter assay was used to determine E2F1 promoter activity. CMV10Ebp1 significantly repressed E2F1 promoter activity by $63 \%$ compared to vector control $(\mathrm{p}<0.001)$. The S363A mutation significantly reversed Ebp1-mediated transcriptional repression $(\mathrm{p}<0.006$, Fig. 4B).

Ebp1 S363A retained the ability to bind the E2F1 promoter. Endogenous Ebp1 is part of a protein complex that binds the E2F1 promoter region of E2F1 regulated genes in vivo (20). This Ebp1-containing complex also binds a synthetic E2F1 promoter oligonucleotide containing two E2F1 consensus motifs. This binding is disrupted when the two E2F1 consensus sites are mutated (20). Since Ebp1 S363A did not repress E2F1 promoter activity as effectively as Ebp1, we performed a DNA affinity assay to test the ability of Ebp1 S363A to bind to the synthetic E2F1 promoter oligo. Lysates of MCF7 cells stably transfected with GFP, GFP-Ebp1 or GFP-Ebp1 S363A were incubated with the biotin labeled E2F1 consensus element oligos immobilized to streptavidin paramagnetic beads. DNA bound proteins were precipitated using magnets, resolved by SDS-PAGE, and analyzed by Western blotting with an antibody to GFP. GFP-Ebp1 was able to interact with the E2F1 consensus oligo, confirming that the GFP tag did not affect Ebp1 binding the promoter element (Fig. 5, lane 7). The S363A mutant was also able to complex to the E2F1 consensus motif, with only a slight loss of binding (Fig. 5, lane 8).

Ebpl S363A did not interact with transcriptional corepressors. The fact that the $\mathrm{S} 363 \mathrm{~A}$ mutant did not significantly repress transcription, but bound the E2F1 promoter element was puzzling. Ebp1 interacts with the transcriptional corepressors HDAC2 and $\mathrm{mSin} 3 \mathrm{a}$ in vitro and in vivo to inhibit 
A



B

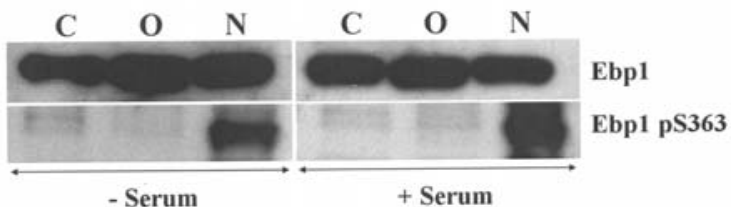

C

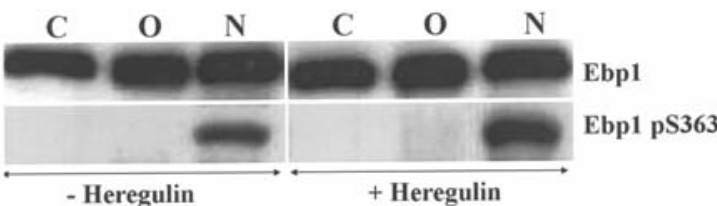

D

I.ight

Field

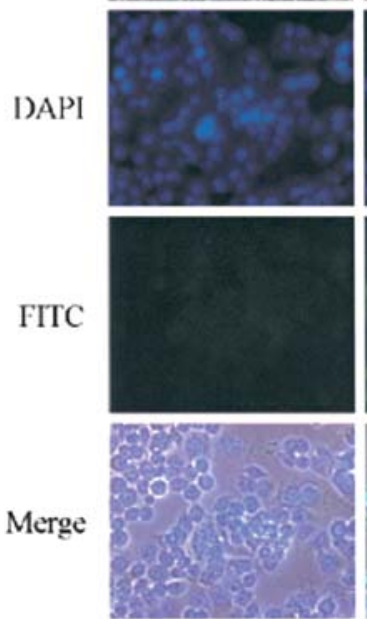

$\operatorname{IgG}$
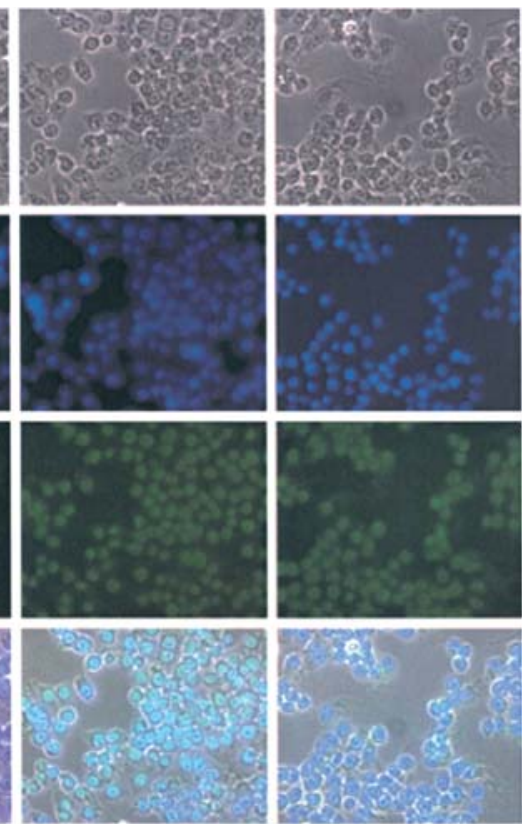

IIDAC

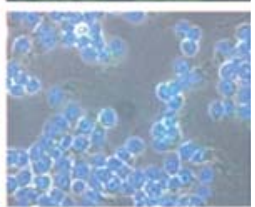

Fbpl pS363

Figure 2. Intracellular distribution of endogenous Ebp1 phosphorylated at S363 under different growth conditions. (A) Efficiency of differential detergent fractionation (DDF) assay. AU565 cell fractions were sequentially isolated using Digitonin (cytoplasm, C), Triton X-100 (organelle, O) and Tween-40/DOC (nuclear, N) extraction buffers as described in the Materials and methods. Fractions were resolved on 10\% SDS gels and probed with antibodies to subcellular compartments: Paxillin (cytoplasm, C), Grp94 (organelle, O) and HDAC2 (nucleus, N). (B) Effect of serum on Ebp1 pS363 localization in AU565 cells. AU565 cells were serum-starved or fed for $48 \mathrm{~h}$ and then fractionated by DDF. Lysates were resolved on $10 \%$ SDS gels and the blots were probed for Ebp1 or pS363-Ebp1 as indicated. (C) Effect of heregulin on Ebp1 pS363 localization in AU565 cells. AU565 cells were serum-starved and then treated or not with heregulin (HRGß1, $10 \mathrm{ng} / \mathrm{ml}$ ) for $1.5 \mathrm{~h}$. Cells were fractionated and lysates resolved on $10 \%$ SDS gels. The blots were probed with antibodies for Ebp1 or pS363-Ebp1. (D) Subcellular localization of endogenous Ebp1 pS363 in logarithmically growing AU565 cells. AU565 cells were plated on glass coverslips at subconfluent densities. On the next day the cells were fixed and immunostained using non-specific rabbit IgG, HDAC2, or pS363-Ebp1 primary antibodies and FITC-conjugated secondary antibodies. DAPI was used to counterstain the nuclei (blue).

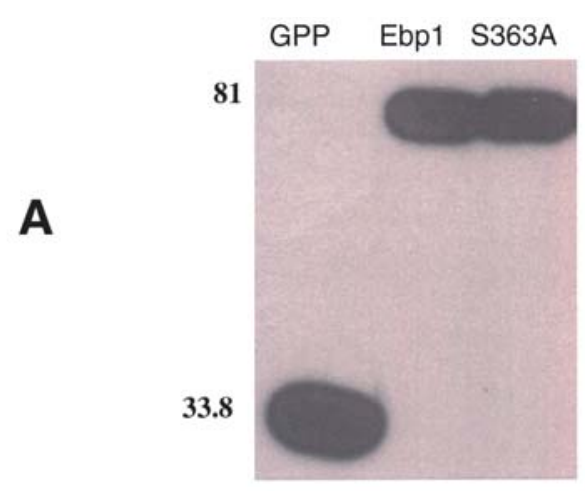

IB: GFP



GFP

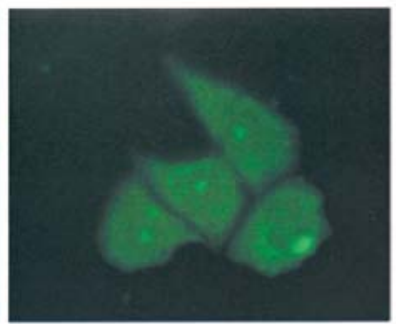

GFP-Ebpl



GFP-Ebpl S363A

Figure 3. Subcellular localization of Ebp1 S363A. (A) Expression of GFP tagged Ebp1 and Ebp1 S363A. MCF7 cells were stably transfected with plasmids expressing GFP, GFP-Ebp1 or GFP-Ebp1 S363A. Cell lysates were resolved by SDS-PAGE and analyzed by Western blotting using a probe for GFP. (B) Subcellular localization of Ebp1 S363A in MCF7 cells. MCF7 cells were stably transfected with plasmids expressing GFP, GFP-Ebp1 and GFP-Ebp1 S363A. The GFP tag was visualized by fluorescence microscopy. 
A

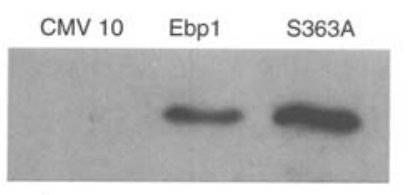

Flag

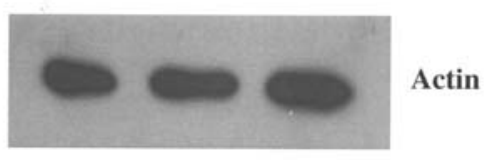

B

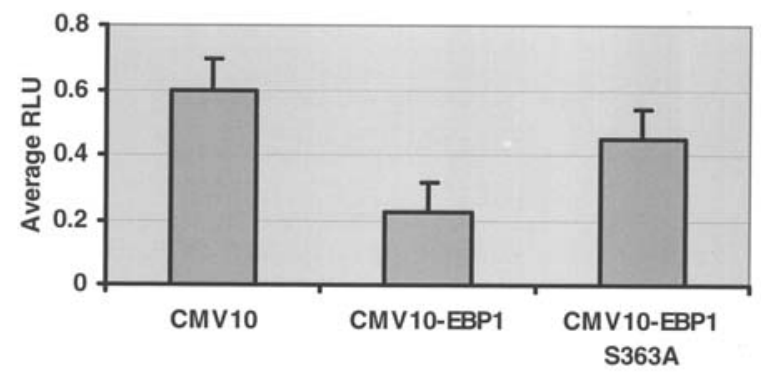

Figure 4. Effect of Ebp1 S363A on E2F1 promoter activity. (A) Expression of Flag tagged Ebp1 mutants. MCF7 cells were transfected with a CMV 10 vector control or plasmids expressing Flag-Ebp1 or Flag-Ebp1 S363A. Cell lysates were resolved by SDS-PAGE and analyzed by Western blotting with antibodies for Flag and Actin as indicated. (B) The S363A mutation partially reverses Ebp1-mediated repression of an E2F1 promoter reporter construct. MCF-7 cells were co-transfected with E2F1-luc, pRL-TK and CMV10, CMV10-Ebp1 or CMV10-Ebp1 S363A. After 48 h, cells were lysed and relative luciferase units were determined as described in the Materials and methods. The data are expressed as relative light units (RLU) which is the ratio of E2F1-luc RLU: pRL-TK RLU for each sample. Each bar represents the mean \pm s.d. of 8 wells. The figure is representative of 3 independent experiments.

\section{U GFP Ebp1 S363A U GFP Ebp1 S363A}

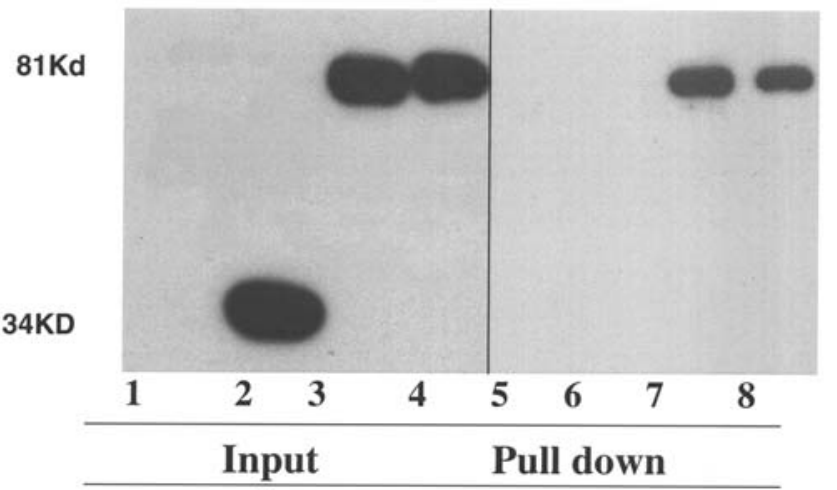

\section{IB: GFP}

Figure 5. Effect of Ebp1 S363A on the formation of E2F1 promoter complexes. MCF7 cells were transfected with plasmids expressing GFP, GFP-Ebp1 or GFP-Ebp1 S363A as described in the Materials and methods. Cell lysates were incubated with a biotin labeled E2F1 consensus element coupled to streptavidin paramagnetic beads. Precipitated proteins were resolved on $10 \%$ SDS gels and analyzed by Western blotting using an antibody to GFP. Left panel, input; right panel, bound proteins.

E2F1-mediated transcription $(19,28)$. We therefore postulated that Ebp1 S363A might not be able to bind these co-repressor proteins. MCF7 cells stably expressing Flag-Ebp1 or FlagEbp1 S363A were lysed and the Flag-tagged proteins were immunoprecipitated using anti-Flag M2 agarose beads. The

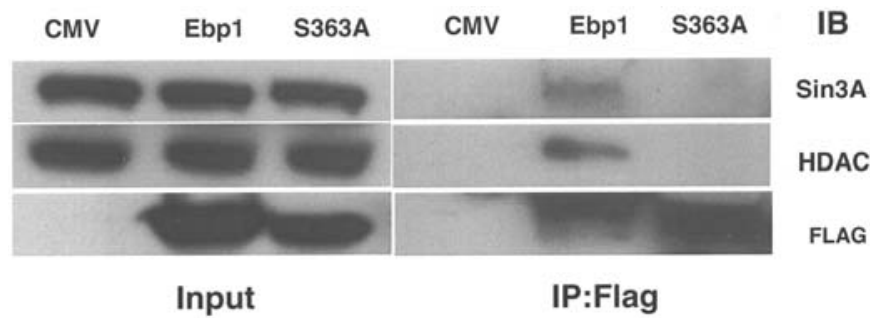

Figure 6. Ebp1 S363A failed to bind mSin3a and HDAC2. MCF7 cells were stably transfected with CMV10 (vector control), CMV10-Ebp1 (expressing FLAG-tagged wild-type Ebp1) or CMV10-Ebp1 S363A (expressing FLAGtagged mutant Ebp1). Cell lysates were incubated with anti-Flag M2 Agarose beads as described in Materials and methods. Immunoprecipitates were resolved on $10 \%$ SDS gels. Western blots were probed with mSin3a, HDAC2 and Flag antibodies as indicated.

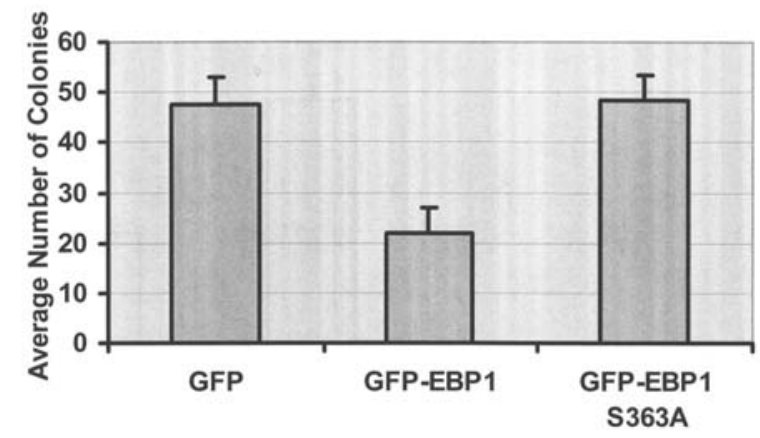

Figure 7. Effect of Ebp1 S363A on MCF7 colony formation. MCF7 cells were transfected with plasmids expressing GFP, GFP-Ebp1 or GFP-Ebp1 S363A as described in the Materials and methods. Cells were selected with G418 for three weeks. Surviving colonies were then fixed and stained with crystal violet and counted. Each bar represents mean \pm s.d. of 6 wells. The data are representative of 3 independent experiments.

immunoprecipitated proteins were resolved by SDS-PAGE and analyzed by Western blotting for interacting proteins. Wild-type Ebp1 bound both mSin3a and HDAC2 as expected. In contrast, Ebp1 S363A was unable to bind either mSin3a or HDAC2 (Fig. 6).

The S363A mutation abrogated the ability of Ebpl to inhibit MCF7 colony formation. Overexpression of Ebp1 inhibits colony formation of breast cancer cells (15). Therefore, a colony forming assay was performed to determine the effects of the S363A mutation on the ability of Ebp1 to inhibit cell growth. MCF7 cells were transfected with GFP, GFP-Ebp1 or GFP-Ebp1 S363A and selected for 3 weeks with G418. As previously shown in Fig. 3A, these proteins are expressed at approximately equal levels in MCF-7 cells. The surviving colonies were then stained and counted. GFP-Ebp1 significantly inhibited MCF7 colony formation by $54 \%(\mathrm{p}<0.0001)$ compared to the vector control. In contrast, the S363A mutant was completely unable to inhibit colony formation (Fig. 7).

\section{Discussion}

Ebp1 is a phosphorylated ErbB3 binding protein with pleiotropic effects. To better understand the biological basis of the multiple effects of Ebp1, we were interested in determining the biological function of its phosphorylated forms. As the $\mathrm{C}$ 
terminal 72 amino acids of Ebp1 are critical to its function, we examined predicted phosphorylation sites within this domain. In this paper, we determined that $\mathrm{S} 363$ is phosphorylated in vivo and that mutation of this site affects Ebp1 function.

We previously demonstrated that Ebp 1 is phosphorylated on Ser and Thr in vivo and that a peptide corresponding to amino acids 361-371 could be phosphorylated by PKC in vitro (23). We therefore generated an antibody to a predicted phosphorylation site (S363) in this region. After testing for the specificity of this antibody, we determined that endogenous Ebp1 was phosphorylated at S363 in vivo. The kinase responsible for the Ser 363 phosphorylation of endogenous Ebp1 has not yet been determined. Treating AU565 cells with the PKC inhibitors Bis and GF109203X did not inhibit Ebp1 phosphorylation at S363 (data not shown). This finding is in keeping with our in vitro data indicating that the S363 site of an Ebp1 peptide corresponding to aa 361-371 was not phosphorylated by PKC in vitro (23). Further, treatment of AU565 cells with HRG, which strongly activates PKC (29), resulted in only a slight increase in phosphorylation at Ser 363 , further supporting the hypothesis that Ser 363 is not phosphorylated by PKC in vivo. Currently studies are being performed in our laboratory to determine the identity of the kinase that phosphorylates S363. In contrast, Ahn et al (17) found that nuclear Ebp1 is phosphorylated by PKC $\delta$ at Ser 360 in response to NGF in PC-12 cells; cytoplasmic Ebp1 is constitutively phosphorylated at this site. Squatrito et al (22) found that the last 94 amino acids of Ebp1 were phosphorylated in vitro by $\mathrm{PKR}$.

In the current study Ebp1 phosphorylated at S363 (Ebp1 pS363) was found exclusively in the nucleus as demonstrated by both biochemical and immunofluorescent methods. These data suggest that Ebp1 pS363 functions in the nucleus and that this site may be important in mediating Ebp1 transcriptional functions and nuclear protein-protein interactions. These studies also suggest that a kinase situated in the nucleus phosphorylates Ebp1 at Ser 363. In contrast, total Ebp1 is distributed ubiquitously in mammalian cells, with a distinct nucleolar localization (16-18). We found in this study that neither serum nor HRG treatment $(1.5 \mathrm{~h})$ changed the subcellular distribution of Ebp1. This is in contrast to our previous data which indicated, by immunohistochemical analysis, that Ebp1 in serum-starved cells was localized exclusively to the cytoplasm and that HRG induced Ebp1 translocation to the nucleus (9). However, those studies were performed with a monoclonal antibody generated to nuclear localized Ebp1 originating from the third ATG (10). It is possible that differences in the antibody or fixation conditions resulted in the differences in subcellular localization.

Although pSer363 Ebp1 was found only in the nucleus, mutation of S363 to alanine did not prevent Ebp1 nuclear localization. Thus, while Ebp1 may become phosphorylated at S363 only in the nucleus, phosphorylation at this site is not required for its nuclear localization. El-Kady and Klenova observed a similar occurrence with the CTCF transcription factor (30). There was no change in the subcellular localization of CTCF containing various single site serine to alanine mutations. Multiple mutations were needed to affect subcellular localization. Thus, phosphoresidues other than S363 may be involved in localizing Ebp1 to the nucleus. For example, Liu et al (24) found that phosphorylation at Ser 360 was needed for Ebp1 nucleolar localization. Alternatively, amino acids with other modifications may be the key to subcellular localization. Squaritto et al (16) found that R364 and K365 were needed for proper nuclear localization of Ebp1.

Ebp1 overexpression results in the repression of transcription of cell cycle regulated genes (18). Using a luciferase reporter under the control of the E2F1 promoter, we found that wild-type Ebp1 repressed luciferase expression compared to the vector control. The S363A mutant significantly decreased Ebp1-mediated transcriptional repression. Similarly, changes in transcriptional activity by mutation of a single amino acid to the non-phosphorylatable alanine have been reported for several transcriptional regulators. For example, the S724A substitution in CTCF converts its function from a transcriptional corepressor to that of a transcriptional activator (30). A S528A substitution in c-myb enhances its activity as a transcriptional activator (31). Our finding suggests that Ebp1 phosphorylated at S363 is needed for optimal E2F1 promoter repression.

Endogenous Ebp1 is part of a protein complex that is assembled on the E2F1 promoter sequence in vitro and in vivo (20). We demonstrate here that GFP-Ebp1 present in cell lysates was able to bind the E2F1 promoter consensus oligo without hindrance from the GFP tag. This interaction is most likely indirect as recombinant wild-type Ebp1 cannot bind to an E2F1 promoter element (20). The S363A mutant was also able to form this complex, although binding was slightly decreased. This slight reduction in DNA binding contrasts with the strong decrease in the ability of mutant Ebp1 to repress E2F1 promoter activity. The fact that Ebp1 S363SA can bind an E2F1 consensus oligo but cannot repress transcription may be due to several factors. First, Ebp1 S363A may bind the oligo under the conditions of substrate excess that are present in the in vitro assay, but not bind the exogenous promoter in vivo. Alternatively, we hypothesized that the composition of a protein complex containing Ebp1 S363A differs from that containing wild-type Ebp1. Indeed, immunoprecipitation analysis indicated that phosphorylation at S363 is needed for Ebp1 to bind either mSin3a or HDAC2, proteins important in transcriptional repression. This finding might explain why Ebp1 can bind an E2F1 consensus element, but cannot repress transcription. In the same vein, Mo et al (32) showed that phosphorylation of the repressor $\mathrm{C} / \mathrm{EBP} \beta$ by Ras causes a change in its activity and interacting partners: when S218 of $\mathrm{C} / \mathrm{EBP} \beta$ is phosphorylated or mutated to glutamic or aspartic acid, it binds the CRSP70-mediator complex turning it into a transcriptional activator. When S218 is not phosphorylated or mutated to alanine, $\mathrm{C} / \mathrm{EBP} \beta$ binds the cdk8-mediator complex becoming a transcriptional repressor .

Ebp1 overexpression results in colony growth inhibition (15). In the current study, wild-type Ebp 1 expression inhibited colony formation by $54 \%$ as compared to vector controls, while Ebp1 S363A was unable to suppress cell growth. Although the ability of Ebp1 to inhibit cell growth was completely abrogated by the alanine mutation, E2F1 transcription was only partially reversed by this mutation. We postulate that the S363A mutation may also affect Ebp1-mediated repression of other E2F1-regulated promoters of cell cycle genes such as cyclin D1, cyclin E and c-myc. The cumulative effect of the partial reversal of Ebp1-mediated repression of multiple genes by the 
S363A mutation may result in complete abrogation of Ebp1's inhibition of cell growth. Future study will examine the effect of Ebp1 S363A on the activity of the cyclin D1, cyclin E and c-myc promoters. Although Ebp1 can inhibit cell growth $(9,16)$, it also suppresses apoptosis (17). The antiapoptotic effects of Ebp1 are also regulated by phosphorylation. In NGFtreated PC12 cells, PKC phosphorylates Ebp1, enhancing Ebp1's antiapoptotic effect by favoring its association with phosphorylated nuclear AKT. Ebp1 S360A is unable to bind nuclear AKT and cells expressing this mutant exhibit DNA fragmentation (17). We have not yet examined the effect of the S363 site on the ability of Ebp1 to prevent apoptosis.

In summary, we determined that phosphorylation of a single serine (S363) affects gene transcription, colony formation and protein binding activities of Ebp1. Further experiments will help us understand how phosphorylation makes Ebp1 a more potent gene repressor through its interacting partners; the results of which will be useful in determining a cancer therapy that targets transcriptional repression.

\section{Acknowledgements}

This study was supported by NIH grants R01 CA76047 and R21 088882-01 and a grant from the Department of Pathology (to AWH). We would like to thank Dr Miriam Smyth for use of the VA fluorescence microscope.

\section{References}

1. Hynes NE and Lane HA: ERBB receptors and cancer: the complexity of targeted inhibitors. Nat Rev Cancer 5: 341-354, 2005.

2. Kraus MH, Issing W, Miki T, Popescu NC and Aaronson SA: Isolation and characterization of ERBB3, a third member of the ERBB/epidermal growth factor receptor family: evidence for overexpression in a subset of human mammary tumors. Proc Natl Acad Sci USA 86: 9193-9197, 1989.

3. Plowman GD, Whitney GS, Neubauer MG, Green JM, McDonald VL, Todaro GJ and Shoyab M: Molecular cloning and expression of an additional epidermal growth factor receptorrelated gene. Proc Natl Acad Sci USA 87: 4905-4909, 1990.

4. Holbro T, Beerli RR, Maurer F, Koziczak M, Barbas CF III and Hynes NE: The ErbB2/ErbB3 heterodimer functions as an oncogenic unit: ErbB2 requires ErbB3 to drive breast tumor cell proliferation. Proc Natl Acad Sci USA 100: 8933-8938, 2003.

5. Olayioye MA, Neve RM, Lane HA and Hynes NE: The ErbB signaling network: receptor heterodimerization in development and cancer. EMBO J 19: 3159-3167, 2000.

6. Lemoine NR, Barnes DM, Hollywood DP, Hughes CM, Smith P, Dublin E, Prigent SA, Gullick WJ and Hurst HC: Expression of the ERBB3 gene product in breast cancer. Br J Cancer 66: 1116-1121, 1992.

7. Koumakpayi IH, Diallo JS, Le PC, Lessard L, Gleave M, Begin LR, Mes-Masson AM and Saad F: Expression and nuclear localization of ErbB3 in prostate cancer. Clin Cancer Res 12: 2730-2737, 2006.

8. Normanno N, Maiello MR and De LA: Epidermal growth factor receptor tyrosine kinase inhibitors (EGFR-TKIs): simple drugs with a complex mechanism of action? J Cell Physiol 194: 13-19, 2003.

9. Yoo JY, Wang XW, Rishi AK, Lessor T, Xia XM, Gustafson TA and Hamburger AW: Interaction of the PA2G4 (EBP1) protein with ErbB-3 and regulation of this binding by heregulin. Br J Cancer 82: 683-690, 2000.

10. Radomski $\mathrm{N}$ and Jost E: Molecular cloning of a murine cDNA encoding a novel protein, p38-2G4, which varies with the cell cycle. Exp Cell Res 220: 434-445, 1995.

11. Horvath BM, Magyar Z, Zhang Y, Hamburger AW, Bako L, Visser RG, Bachem CW and Bogre L: EBP1 regulates organ size through cell growth and proliferation in plants. EMBO J 25 : 4909-4920, 2006.
12. Xia X, Lessor TJ, Zhang Y, Woodford N and Hamburger AW: Analysis of the expression pattern of Ebp1, an ErbB-3-binding protein. Biochem Biophys Res Commun 289: 240-244, 2001.

13. Lamartine J, Seri M, Cinti R, Heitzmann F, Creaven M, Radomski N, Jost E, Lenoir GM, Romeo G and Sylla BS: Molecular cloning and mapping of a human cDNA (PA2G4) that encodes a protein highly homologous to the mouse cell cycle protein p38-2G4. Cytogenet Cell Genet 78: 31-35, 1997.

14. Zhang Y, Wang XW, Jelovac D, Nakanishi T, Yu MH, Akinmade D, Goloubeva O, Ross DD, Brodie A and Hamburger AW: The ErbB3-binding protein Ebp1 suppresses androgen receptor-mediated gene transcription and tumorigenesis of prostate cancer cells. Proc Natl Acad Sci USA 102: 9890-9895, 2005

15. Lessor TJ, Yoo JY, Xia X, Woodford N and Hamburger AW: Ectopic expression of the ErbB-3 binding protein ebp1 inhibits growth and induces differentiation of human breast cancer cell lines. J Cell Physiol 183: 321-329, 2000.

16. Squatrito M, Mancino M, Donzelli M, Areces LB and Draetta GF: EBP1 is a nucleolar growth-regulating protein that is part of pre-ribosomal ribonucleoprotein complexes. Oncogene 23: 4454-4465, 2004.

17. Ahn JY, Liu X, Liu Z, Pereira L, Cheng D, Peng J, Wade PA, Hamburger AW and Ye K: Nuclear Akt associates with PKCphosphorylated Ebp1, preventing DNA fragmentation by inhibition of caspase-activated DNase. EMBO J 25: 2083-2095, 2006.

18. Xia X, Cheng A, Lessor T, Zhang Y and Hamburger AW: Ebp1, an ErbB-3 binding protein, interacts with $\mathrm{Rb}$ and affects $\mathrm{Rb}$ transcriptional regulation. J Cell Physiol 187: 209-217, 2001.

19. Zhang YX, Woodford N, Xia XM and Hamburger AW: Repression of E2F1-mediated transcription by the ErbB3 binding protein Ebp1 involves histone deacetylases. Nucleic Acids Res 31: 2168-2177, 2003 .

20. Zhang Y and Hamburger AW: Heregulin regulates the ability of the ErbB3-binding protein Ebp1 to bind E2F promoter elements and repress E2F-mediated transcription. J Biol Chem 279: 26126-26133, 2004.

21. Bose SK, Sengupta TK, Bandyopadhyay S and Spicer EK: Identification of Ebp 1 as a component of cytoplasmic bcl-2 mRNP (messenger ribonucleoprotein particle) complexes. Biochem J 396: 99-107, 2006.

22. Squatrito M, Mancino M, Sala L and Draetta GF: Ebp1 is a dsRNA-binding protein associated with ribosomes that modulates eIF2alpha phosphorylation. Biochem Biophys Res Commun 344: 859-868, 2006.

23. Lessor TJ and Hamburger AW: Regulation of the ErbB3 binding protein Ebp1 by protein kinase C. Mol Cell Endocrinol 175: 185-191, 2001.

24. Liu Z, Ahn JY, Liu X and Ye K: Ebp1 isoforms distinctively regulate cell survival and differentiation. Proc Natl Acad Sci USA 103: 10917-10922, 2006.

25. Ramsby ML and Makowski GS: Differential detergent fractionation of eukaryotic cells. Analysis by two-dimensional gel electrophoresis. Methods Mol Biol 112: 53-66, 1999.

26. Johnson DG, Ohtani K and Nevins JR: Autoregulatory control of E2F1 expression in response to positive and negative regulators of cell cycle progression. Genes Dev 8: 1514-1525, 1994.

27. Jans DA and Hubner S: Regulation of protein transport to the nucleus: central role of phosphorylation. Physiol Rev 76: 651-685, 1996.

28. Zhang Y, Akinmade D and Hamburger AW: The ErbB3 binding protein Ebp1 interacts with Sin3A to repress E2F1 and ARmediated transcription. Nucleic Acids Res 33: 6024-6033, 2005.

29. Emkey R and Kahn CR: Cross-talk between phorbol estermediated signaling and tyrosine kinase proto-oncogenes. I. Activation of protein kinase $\mathrm{C}$ stimulates tyrosine phosphorylation and activation of ErbB2 and ErbB3. J Biol Chem 272: 31172-31181, 1997.

30. El-Kady A and Klenova E: Regulation of the transcription factor, CTCF, by phosphorylation with protein kinase CK2. FEBS Lett 579: 1424-1434, 2005.

31. Miglarese MR, Richardson AF, Aziz N and Bender TP: Differential regulation of c-Myb-induced transcription activation by a phosphorylation site in the negative regulatory domain. $\mathrm{J}$ Biol Chem 271: 22697-22705, 1996.

32. Mo X, Kowenz-Leutz E, Xu H and Leutz A: Ras induces mediator complex exchange on C/EBP beta. Mol Cell 13: 241-250, 2004. 